\title{
IMPACTO DEL MODELO DE GESTIÓN PÚBLICA MUNICIPAL EN LAS DESIGUALDADES TERRITORIALES DE GUAYAQUIL
}

\section{IMPACT OF THE MUNICIPAL PUBLIC MANAGEMENT MODEL IN THE TERRITORIAL INEQUALITIES OF GUAYAQUIL}

José Fernando Romero Subia, Ing. Ingeniero Comercial (Ecuador). Analista de Customer Care en Conecel S.A., Ecuador. jfernandoromero1984@gmail.com

María Salomé Ochoa Rico, Ing. Ingeniera en Marketing (Ecuador). Coordinadora de Planificación en el Municipio de Guayaquil, Ecuador. salomeochoa@hotmail.com

Econ. Miguel Alexander Coloma Suárez Economista (Ecuador). Analista de Planificación e Inversión en el Ministerio de Producción, Comercio Exterior, Inversiones y Pesca, Ecuador. migcoloma@mgs.ecotec.edu.ec

Econ. Stefania Carolyn Baidal Chang Economista (Ecuador). Jefa de Presupuesto en el Municipio de San Jacinto de Yaguachi, Ecuador. stefi_moises@hotmail.com

\section{ARTÍCULO DE INVESTIGACIÓN}

Recibido: 16 de septiembre de 2019.

Aceptado: 22 de noviembre de 2019. 


\section{RESUMEN}

Este trabajo tiene como objetivo medir el impacto de la administración pública de Guayaquil en el ámbito de las desigualdades territotriales de la ciudad, para esto se analizaron las variables clínicas móviles, impuestos prediales, tasas de habilitación, metros cuadrados de áreas verdes por habitante y cobertura del agua potable consideradas iniciativas que aportan a la disminución de la desigualdad, el período analizado comprende desde 2007 al 2018 con datos extraídos de las paginas oficiales del Banco Central del Ecuador, Municipio de Guayaquil e Interagua. El procedimiento metodológico utilizado se basa en un enfoque cuantitativo a través de la elaboración de un análisis econométrico de un modelo empírico de regresión lineal utilizando como variable dependiente la desigualdad de ingresos (medido por el coeficiente de Gini) en la ciudad de Guayaquil, finalmente, se pudo dar observancia y validez del impacto de una de las variables propuestas en la disminución de la desigualdad de ingresos de la ciudad de Guayaquil, como fue el caso de la variable independiente Clínicas Móviles (MOV), a diferencia de las otras variables propuestas, las cuales presentaron problemas de colinealidad.

Palabras clave: Desigualdad económica territorial, territorio, administración pública, salud pública

\section{ABSTRACT}

The objective of this paper is to measure the impact of the public administration of Guayaquil in the field of territotrial inequalities of the city, for this we analyzed the mobile clinical variables, property taxes, habilitation rates, square meters of green areas per inhabitant and drinking water coverage considered initiatives that contribute to the reduction of inequality, the period analyzed includes from 2007 to 2018 with data extracted from the official pages of the Banco Central del Ecuador, Municipio de Guayaquil e Interagua. The methodological procedure used is based on a quantitative approach through the elaboration of an econometric analysis of an empirical linear regression model using as a dependent variable income inequality (Gini coefficient) in the city of Guayaquil, finally, It was possible to observe and validate the impact of one of the variables proposed in the reduction of income inequality in the city of Guayaquil, as was the case of the independent variable Mobile Clinics (MOV), unlike the other variables proposed, which presented collinearity problems. 
Keywords: Territorial economic inequality, territory, public administration, public health

\section{INTRODUCCIÓN}

Las dimensiones de cambio han evolucionado, pasando del modelo fordista y keynesiano de bienestar a un modelo emergente que busca superar los límites de la desigualdad y exclusión social. En el contexto de la globalización, los cambios producidos por los impactos tecnológicos, el avance de la ciencia y el desarrollo de la conciencia social impulsada por líderes políticos, sociales y ONG's., han promovido en las regiones la implementación de acciones orientadas a disminuir los índices de desigualdad.

Entonces, para obtener una visión más clara sobre el impacto producido por los índices de desigualdad es importante considerar el análisis de indicadores que midan las diferencias entre las sociedades, con base en la implementación de políticas dirigidas a la inclusión y mejoramiento de la calidad de vida de la población.

En América Latina, debido a las limitaciones presupuestarias, las diferencias estructurales entre países, así como la creciente crisis económica política y social que caracteriza a la región, han generado un notable incremento en los índices de desigualdad.

En el Caso de Ecuador, se ha evidenciado una leve disminución de la pobreza extrema a consecuencia de las políticas dirigidas al incremento del gasto social implementadas en los últimos años. Sin embargo, las brechas de desigualdad entre segmentos socioeconómicos, así como entre sectores rurales y urbanos han ido incrementando.

En ciudades de mayor extensión territorial, como es el caso de Guayaquil, se registran niveles altos de desigualdad social y económica a consecuencia del incremento poblacional debido a las corrientes migratorias tanto a nivel nacional e incluso internacional, lo cual ha generado un incremento en la población que habita en sectores urbano marginales, muchos de estos carentes de acceso a servicios básicos. 
De este modo, el presente trabajo de investigación busca determinar cuál es el impacto de la gestión municipal en la disminución del índice de desigualdad medido por el coeficiente GINI en el cantón Guayaquil.

Asimismo, partiendo de la idea de la importancia de considerar estrategias de política pública municipal que permitan buscar formas eficientes de disminuir los impactos negativos generados por la desigualdad, se han planteado los siguientes objetivos general: determinar la contribución de la Gestión Municipal en la disminución de la desigualdad medido por el coeficiente de GINI en el cantón Guayaquil. Así como los siguientes objetivos específicos: sistematizar aspectos teóricos relacionados al incremento del índice de desigualdad y diagnosticar la contribución de la gestión municipal en la disminución del índice de desigualdad en el cantón Guayaquil.

Por otra parte, el procedimiento metodológico que determina el diseño de este trabajo consiste en un enfoque cuantitativo, a partir de un análisis econométrico que mide a lo largo de una serie de tiempo los efectos generados por los cambios en las variables involucradas, lo cual permitirá conocer la incidencia de dichas variables en el índice de desigualdad a escala local.

De igual forma, el método utilizado en el desarrollo de este trabajo tiene un alcance de tipo exploratorio/experimental, dentro de un análisis que parte de la búsqueda de premisas particulares con miras a establecer conclusiones generales.

En consecuencia, luego de un análisis de la teoría y la práctica desarrollada a lo largo de este trabajo, los principales resultados alcanzados demuestran una relación inversamente proporcional entre el índice de desigualdad GINI y el incremento de la cobertura del servicio de salud proporcionado por el gobierno local, evidenciando un mejoramiento en la calidad de vida de la población estudiada, plasmado en la disminución del índice GINI a medida que incrementa la cobertura en salud.

\section{REVISIÓN TEÓRICA}

Debido a los problemas económicos, culturales, políticos y sociales que se presentan diariamente dentro de un territorio no es posible plantear una conceptualización que califique a un territorio 
únicamente como un simple espacio físico, sino mas bien como el resultado de acontecimientos y hechos históricos de una sociedad que busca la satisfacción de sus necesidades a través del mejoramiento de los sistemas productivos, respondiendo así a los desafíos de la globalización, logrando competir en un mercado cambiante que necesita de los principios de justicia, accesibilidad y equidad para contrarrestar las desigualdades.

Por tanto, debido a la creciente tendencia globalizadora, así como la búsqueda del desarrollo del capital intelectual, el territorio pasa a convertirse de un lugar físico para ser habitado, a ser considerado un espacio dónde se despliegan actividades productivas y estratégicas que busca el desarrollo y mejoramiento de las prácticas empresariales a través de la implementación de planes innovadores con la acción de instituciones públicas locales con miras a promover una eficiente gestión social y distribución de ingresos (Garofoli, 1986).

Así mismo, desde un punto de vista de carácter político, se considera al territorio como un espacio construido socialmente, a partir de las experiencias, conflictos y acuerdo de los grupos de poder que habitan en el territorio, es decir, constituye un constructo social que se transforma conforme a los intereses de quien lo gobierna, generando de esta manera territorios centrales, y periféricos lo cual conlleva a un incremento de los índices de desigualdad de ingresos (Muñoz, 2015).

De este modo, Vásquez (1999) define al territorio como un agente de transformación donde es necesario promover el aprovechamiento de los recursos productivos y la generación de actividades económicas para lograr una adecuada e incluyente interacción entre los agentes del desarrollo con todas las clases sociales, creando dinamismo económico, orientado a disminuir los índices de desigualdad.

Por otra parte, Stiglitz (2012), asegura que la desigualdad es un efecto de las desacertadas decisiones políticas en un territorio, antes que una consecuencia económica. El catedrático establece un análisis de la desigualdad a nivel mundial donde concluye que el $1 \%$ de la población más rica, posee el $99 \%$ de la riqueza mundial, y asegura que los hijos de padres millonarios, algunos de ellos con bajos rendimientos académicos, tienen mayor probabilidad de convertirse en adultos adinerados que los jóvenes con altos rendimientos académicos descendientes de padres pobres. 
El premio Nobel (Stiglitz, 2012) apuesta a la disminución de la desigualdad por medio de una adecuada implementación de políticas estatales intervencionistas, aduciendo que los mercados por si solos no son eficientes ya que proporcinan ventajas a los más ricos, generando problemas sociales tales como altos índices de criminalidad, disminución de la esperanza de vida, niveles bajos de calidad en educación, inseguridad, en consecuencia, baja la calidad de vida, etc.

Además, (Stiglitz, 2012), expone que la disminución del gasto público es una forma errónea de contarrestar las crisis económicas, y que la única forma de hacer funcionar esa política de austeridad es teniendo socios comerciales con economías sólidas, lo cual permitirá fortalecer las exportaciones para generar desarrollo, y disminuir el impacto de la disminución del gasto social. Por tanto, sugiere que la desigualdad de ingresos proviene de la inequidad salarial, y de la riqueza derivada del capital y la acumulación de los patrimonios familiares. Sin embargo, para otros autores como (Boisier, 1988), la transformación económica y social que se genera dentro de un territorio debe ser el resultado del desarrollo de las capacidades del capital humano, promovido por los actores políticos, tales como gobiernos centrales y seccionales, líderes sociales, sector empresarial, entre otros, quiene ejercen acciones dirigidas a crear crecimiento económico con responsabilidad social de modo que se vean disminuidos los índices de desigualdad, fortaleciendo la inclusión de la clase media y baja.

Por lo que, construir socialmente una región, superando los conflictos generados por la desigualdad, conlleva a la acción de los actores locales en la búsqueda de lograr potenciar su capacidad de auto-organización, transformando una comunidad inanimada, segmentada por intereses sectoriales, y en definitiva pasiva, en otra, organizada, cohesionada, capaz de transformarse en sujeto de su propio desarrollo (Boisier, 1988).

De otra manera, es posible relacionar también la desigualdad generada en los territorios con el avance de la globalización, lo cual ha promovido a más de la integración de los mercados, una intensificación de los paradigma sociales, logrando crear diferencias estructurales dentro de las sociedades, generando desigualdades de ingresos lo cual se convierte, para los grupos vulnerables, en falta de acceco a los servicios básicos y escazas oportunidades para laborar en empleos dignos con salarios justos (Castells, 1998). 
Por consiguiente, (Veiga \& Rivoir, 2004) destacan que las fragmentaciones sociales se derivan de la diferenciación que se presenta en los territorios debido a una inequitativa distribución de las riquezas a través de la formación de las clases sociales.

En general, la desigualdad llega a ser una preocupación vivencial, la cual a medida en que un país se desarrolla y va generando ingreso, dicho ingreso con el paso del tiempo empieza a concentrarse en determinados sectores socioeconómicos como resultado de una mala distribución.

Actualmente, existe un indicador que mide la desigualdad de ingresos, denominado coeficiente de Gini, cuyos valores se ubican entre 0 y 1 . Significando 0 total igualdad y 1 total desigualdad y cuyo cálculo nos da una idea de los graves problemas de desigualdad que atraviesa la sociedad, reflejados en indicadores que señalan el aumento del subempleo y la informalidad laboral, entre otros.

Entonces, los efectos de la producción capitalista y su manifestación en la globalización sentencian las condiciones de los territorios, por lo tanto, como una alternativa para disminuir de alguna manera la desigualdad social y de ingresos, surge desde una perspectiva territorial, el desarrollo a partir del territorio (Alemán, A., 2019)

Entre tanto, al referirnos a la desigualdad como consecuencia de las malas decisiones a nivel de política pública, siendo los gobiernos los principales gestores de desarrollo y agentes promovedores de la disminución de la desigualdad de ingresos, señalamos al padre fundador de la ciencia de la administración pública, (Bonnin, 1812) quien define a esta, como la gestión de los asuntos de las personas, bienes y acciones ciudadanas como parte de un Estado que se involucra y que busca la armonía en las relaciones humanas dentro de una colectividad.

De forma similar, (Stein, 1897) define a la administración pública como una actividad del Estado que tiene su base en los órganos estatales, alineado con la interpretación económica y los conceptos como proletariado y lucha de clases, promoviendo la búsqueda de un estado que supere el interés por los beneficios particulares y clasistas que generan desigualdad y crean desorden y choques de carácter social. 
Por otra parte (Marx, 1968) define a la gestión pública como la actividad que organiza el Estado; en donde las negociaciones internacionales, comercio y actividades de carácter privada se ven en la necesidad de ser reguladas por la presencia de la administración pública.

En consecuencia, es imperativo que el ser humano logre desarrollarse en un territorio que le garantice participación en los procesos de producción de sus recursos, así como también que genere a través de regulaciones una eficiente administración pública, y distribución de ingresos además de una adecuada implementación de acciones que sean ejecutadas con responsabilidad social, y que estén bajo los principios de equidad, justicia y sostenibilidad.

Entonces, tal como lo plantea (Correa, 2016) el desarrollo de las regiones, involucra el desarrollo de una identidad cultural progresista y participativa, siempre y cuando dicha identidad conlleve a lograr mayor eficiencia en la distribución de los ingresos.

Finalmente, cabe señalar que la creciente tendencia a generar procesos de descentralización se ha convertido en un mecanismo que promueve la participación activa de los actores propios del territorio en la identificación de sus necesidades y en la búsqueda de los elementos que contribuyan a generar desarrollo y a la disminución de la desigualdad de ingresos, a través del fortalecimiento de las competencias asignadas a los gobiernos locales (COOTAD, 2010)

Por consiguiente, el éxito o fracaso de las estrategias encaminadas a disminuir los índices de desigualdad, con base en los procesos de descentralización, depende mayormente de la capacidad que tienen los líderes políticos y sociales de identificar y priorizar las necesidades de la población y de encaminarlas de forma acertada hacia la implementación de procesos de desarrollo productivos, de modo que generen bienestar y equidad de ingresos.

Entonces, desde la perspectiva del desarrollo humano, como base de la administración pública, es una opción viable y esperanzadora al colocar al ser humano en el centro de atención de toda política pública, encaminada a generar equidad y a restituir la dignidad como persona, convirtiéndolos en sujetos y objetos del desarrollo (Casas, 2018, p. 227) 


\section{ANÁLISIS DEL CASO PROPUESTO}

La transferencia de competencias y recursos provenientes del gobierno central hacia los gobiernos seccionales, como resultado de las políticas de descentralización implementadas en los últimos años, ha marcado el punto de partida hacia la construcción de un nuevo reto por parte de los líderes políticos locales, quienes desde hace un tiempo atrás son los encargados de articular acciones de manera autónoma, eficiente y equitativa en favor del bienestar social, e igualdad económica. (Vázquez, 2015)

El objetivo principal de los Gobiernos locales, está enfocado en promover la evolución de los procesos económicos territoriales como método de solución para lograr la satisfacción de las necesidades de los habitantes del territorio. (Molina, 2015). Dicho esto, el Gobierno Autónomo Descentralizado, Municipal de Guayaquil en pro de fortalecer el bienestar y de contribuir a la disminución del índice de desigualdad en el cantón Guayaquil, ha implementado entre sus política públicas acciones dirigidas a generar igualdad. Las cuales se detallan a continuación:

$\checkmark \quad$ Implementación de servicio de salud a través de clínicas móviles (MOV)

$\checkmark \quad$ Cobro de impuestos prediales con el fin de generar ingresos económico para invertir en gasto social (PREDIAL)

$\checkmark \quad$ Cobro de Tasas de Habilitación a locales comerciales, de modo que incremente el ingreso de la ciudad. (HAB)

$\checkmark \quad$ Metros cuadrados de espacio de áreas verdes por habitante para el fomento de lugares recreacionales (VERDE)

$\checkmark \quad$ Incremento en la capacidad de cobertura en el servicio agua potable y alcantarillado (AGUA)

La finalidad del presente trabajo de investigación es analizar la variabilidad en la desigualdad de los ingresos de los habitantes en el cantón Guayaquil, representado por el coeficiente de Gini, el cual mide los niveles de desigualdad y que será identificado con la variable dependiente (Y) en el modelo empírico propuesto, con respecto a las variables explicativas $(\mathbf{X})=\mathbf{M O V}$, AGUA,PREDIAL,HAB,VERDE. 
Se ha escogido como variable dependiente la desigualdad de ingresos (medido por el coeficiente de Gini) en la ciudad de Guayaquil, para corroborar la literatura que plantea el vínculo existente entre desigualdad y tasa de crecimiento, por consiguiente proponemos un modelo empírico de regresión lineal que describe la importancia de disminuir la desigualdad a través de las variables explicativas que detallo a continuación:

- $\quad$ Clínicas móviles (MOV): Considerado por ser un indicador de desarrollo humano (IDH), por lo cual concluimos que el proveer acceso gratuito a la salud implica disminución de la desigualdad debido a que los ciudadanos con pocos ingresos no tendrán la necesidad de dirigir sus escasos recursos económicos al pago del servicio de salud privada. El Municipio de Guayaquil, a través del programa Clínicas Móviles atienden anualmente 20160 pacientes por clínica, teniendo en la actualidad 36 clínicas móviles al servicio de la ciudadanía.

- $\quad$ Acceso al agua y alcantarillado (AGUA): Se considera que mayor cobertura en la prestación de este servicio, generará un impacto positivo que conlleve a la disminución de la desigualdad.

- Prediales (PREDIAL) y tasa de habilitación(HAB): Debido a que la implementación de una adecuada política fiscal influye en el crecimiento económico, concluimos que la mayor recaudación de tributos permitirá incrementar el presupuesto municipal el cual es destinado en un $90 \%$ a obra pública y gasto social, de esta manera contribuyendo así con la disminución de la desigualdad.

- $\quad$ Áreas verdes (VERDE): Por ser un factor clave para el cuidado de la salud de la población, debido a que actúan como pulmones que renuevan el aire polucionado, al tiempo que relajan el ambiente de ciudades llenas de contaminación y hormigón, consideramos que el incremento de áreas verdes influye en el bienestar. Por este motivo es crucial incorporar dicha variable como un factor explicativo del modelo de regresión planteado.

El incremento en la cobertura por parte del Gobierno Autónomo Descentralizado Municipal de Guayaquil en servicios de salud, por medio de clínicas móviles, el aumento en la recaudación de impuestos prediales y del tributo de Tasa de Habilitación, así como la ampliación en la cobertura del servicio de agua potable y alcantarillado, además del aumento de espacios verdes por 
habitante, disminuyen el índice de desigualdad medido por el coeficiente de GINI en el cantón Guayaquil.

Cuadro 1. Datos

\begin{tabular}{|c|c|c|c|c|c|c|}
\hline ANIO & GINI & PREDIAL & HAB & AGUA & MOV & VERDE \\
\hline 2007 & 0,51 & 12121633,2 & 948019,32 & 2235683,15 & 80640 & 6 \\
\hline 2008 & 0,4 & 13121906,07 & 1243621,66 & 2270252,7 & 201600 & 6,15 \\
\hline 2009 & 0,43 & 11661501,1 & 2220271,77 & 2304822,26 & 322560 & 6,22 \\
\hline 2010 & 0,4 & 16043671,22 & 2413550,01 & 2342273 & 322560 & 6,3 \\
\hline 2011 & 0,4 & 16986221,99 & 1438076,84 & 2373042 & 322560 & 6,52 \\
\hline 2012 & 0,35 & 17581904,27 & 4327666,49 & 2402266 & 564480 & 7,7 \\
\hline 2013 & 0,42 & 20000000 & 5684874,6 & 2431078 & 564480 & 8,26 \\
\hline 2014 & 0,36 & 24000000 & 7089396,4 & 2459410 & 564480 & 24,58 \\
\hline 2015 & 0,37 & 24600000 & 8367377,39 & 2487213 & 725760 & 24,58 \\
\hline 2016 & 0,38 & 26400000 & 8554573,64 & 2514446 & 725760 & 25,27 \\
\hline 2017 & 0,4 & 26800000 & 8330750,71 & 2541115,35 & 725760 & 25,61 \\
\hline 2018 & 0,39 & 27200000 & 9115242,76 & 2573669,59 & 725760 & 25,96 \\
\hline
\end{tabular}

Fuente: Elaboración Propia a partir del Banco Central del Ecuador (GINI), Municipalidad de Guayaquil (PREDIAL-HAB-MOV-VERDE), Interagua (AGUA)

\section{El modelo inicial se representa de la siguiente manera:}

\section{Gini $=$ $\beta O+\beta 1 M O V+\beta 2 P R E D I A L+\beta 3 H A B+\beta 4 V E R D E+\beta 5 A G U A$}

Debido a que las variables independientes propuestas se expresan en diferentes magnitudes, hemos considerado analizar su variabilidad de forma porcentual tomando como base el modelo $\log$ - log, de la siguiente manera:

$\ln ($ Gini $)=\$ 0+\beta 1 \ln (\mathrm{MOV})+\beta 2 \ln ($ PREDIAL) + $33 \ln (\mathrm{HAB})+\beta 4 \ln (\mathrm{VERDE})+\beta 5 \ln (\mathrm{AGUA})$

Una vez determinado el modelo funcional, se procede a ejecutar la regresión y comprobar si el modelo es consistente, es decir si hay relación entre el fenómeno estudiado (niveles de desigualdad) y cada una las variables explicativas.

Call:

Im(formula $=$ DATO\$LGINI -1 + DATO\$LPREDIAL + DATO\$LHAB + DATO\$LAGUA + DATO\$LMOV + DATO\$LVERDE) 


\section{Residuals:}

\begin{tabular}{|c|c|c|c|c|}
\hline Min & 1Q & Median & 3Q & Max \\
\hline-0.09251 & -0.02390 & 0.01161 & 0.04012 & 0.06406 \\
\hline
\end{tabular}

\section{Coefficients:}

\begin{tabular}{|l|c|c|c|c|}
\cline { 2 - 5 } \multicolumn{1}{c|}{} & Estimate & Std. Error & t value & $\operatorname{Pr}(>|t|)$ \\
\hline DATOSLPREDIAL & -0.04289 & 0.22922 & -0.187 & 0.8569 \\
\hline DATOSLHAB & 0.12225 & 0.09764 & 1.252 & 0.2508 \\
\hline DATOSLAGUA & 0.06365 & 0.22618 & 0.281 & 0.7865 \\
\hline DATOSLMOV & -0.22517 & 0.09141 & -2.463 & $0.0433^{*}$ \\
\hline DATO\$LVERDE & -0.03080 & 0.08810 & -0.350 & 0.7369 \\
\hline
\end{tabular}

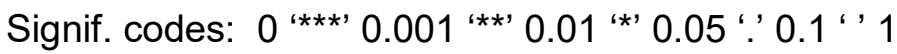

Residual standard error: 0.06647 on 7 degrees of freedom

Multiple R-squared: 0.997, Adjusted R-squared: 0.9948

F-statistic: 464 on 5 and 7 DF, p-value: 1.154e-08

Los resultados expuestos en la ejecución del modelo no muestran un buen grado de consistencia, motivo por el lo que se procede a realizar la prueba VIF para comprobar si existe colinealidad entre variables, los resultados obtenidos se muestran a continuación:

MODELO<Im(DATO\$LGINI 1+DATO\$LPREDIAL+DATO\$LHAB+DATO\$LAGUA+DATO\$LMOV +DATO\$LVERDE)vif(MODELO)

\begin{tabular}{|c|c|c|c|c|}
\hline DATOSLPREDIAL & DATO\$LHAB & DATO\$LAGUA & DATO\$LM0V & DATO\$LVERDE \\
\hline 40058.8192 & 5956.1298 & 29999.7119 & 3808.3792 & 136.0293 \\
\hline
\end{tabular}

MODELO<-Im(DATO\$LGINI -1+DATO\$LPREDIAL+DATO\$LHAB) $\operatorname{vif(MODELO)}$

\begin{tabular}{|c|c|}
\hline DATO\$LPREDIAL & DATO\$LHAB \\
\hline 713.196 & 713.196 \\
\hline
\end{tabular}

MODELO<-Im(DATO\$LGINI -1+DATO\$LPREDIAL+DATO\$LAGUA) vif(MODELO) 


\begin{tabular}{|c|c|}
\hline DATO \$LPREDIAL & DATO\$LAGUA \\
\hline 4305.59 & 4305.59 \\
\hline
\end{tabular}

MODELO<-Im(DATO\$LGINI -1+DATO\$LPREDIAL+DATO\$LMOV) vif(MODELO)

\begin{tabular}{|c|c|}
\hline DATO\$LPREDIAL & DATO\$LMOV \\
\hline 797.9469 & 797.9469 \\
\hline
\end{tabular}

MODELO<-Im(DATO\$LGINI -1+DATO\$LPREDIAL+DATO\$LVERDE) vif(MODELO)

\begin{tabular}{|c|c|}
\hline DATO\$LPREDIAL & DATO\$LVERDE \\
\hline 16.83249 & 16.83249 \\
\hline
\end{tabular}

MODELO<-Im(DATO\$LGINI -1+DATO\$LAGUA+DATO\$LHAB) vif(MODELO)

\begin{tabular}{|c|c|}
\hline DATO\$LAGUA & DATO $\$$ LHAB \\
\hline 391.5487 & 391.5487 \\
\hline
\end{tabular}

MODELO<-Im(DATO\$LGINI -1+DATO\$LAGUA+DATO\$LMOV) vif(MODELO)

\begin{tabular}{|c|c|}
\hline DATO\$LAGUA & DATO\$LMOV \\
\hline 456.5944 & 456.5944 \\
\hline
\end{tabular}

MODELO<-Im(DATO\$LGINI -1+DATO\$LAGUA+DATO\$LVERDE) vif(MODELO)

\begin{tabular}{|c|c|}
\hline DATO\$LAGUA & DATO\$LVERDE \\
\hline 15.11876 & 15.11876 \\
\hline
\end{tabular}

MODELO<-Im(DATO\$LGINI -1+DATO\$LHAB+DATO\$LMOV) vif(MODELO)

\begin{tabular}{|c|r|}
\hline DATO\$LHAB & DATO\$LMOV \\
\hline 2447.197 & 2447.197 \\
\hline
\end{tabular}

MODELO<-Im(DATO\$LGINI -1+DATO\$LHAB+DATO\$LVERDE) vif(MODELO)

\begin{tabular}{|c|c|}
\hline DATO\$LHAB & DATO\$LVERDE \\
\hline 21.50737 & 21.50737 \\
\hline
\end{tabular}


MODELO<-Im(DATO\$LGINI -1+DATO\$LMOV+DATO\$LVERDE)

vif(MODELO)

\begin{tabular}{|c|c|}
\hline DATO\$LMOV & DATO\$LVERDE \\
\hline 19.46658 & 19.46658 \\
\hline
\end{tabular}

La prueba VIF nos indica que existe multicolinealidad entre variables. Dicho esto hemos procedido a evaluar cada variable por separado y los resultados obtenidos son:

Cuadro 2. Modelo.

\begin{tabular}{|c|c|c|c|c|c|c|}
\hline MODELO & ESTIMATE & STD. ERROR & T. VALUE & Pr & R - SQUARED & P- VALUE \\
\hline $\log (\mathrm{GINI})=\beta 0-\beta 1 \log (\mathrm{MOV})+\mathrm{E}$ & -0.07128 & 0.00151 & -47.2 & $4.73 \mathrm{E}-14$ & 0.9951 & $4.73 \mathrm{E}-14$ \\
\hline $\log (\mathrm{GINI})=\beta 0-\beta 1 \log (\mathrm{PREDIAL})+\mathrm{E}$ & -0.055012 & 0.001542 & -35.68 & $1.01 \mathrm{E}-12$ & 0.9914 & $1.01 \mathrm{E}-12$ \\
\hline $\log (\mathrm{GINI})=\beta 0-\beta 1 \log (\mathrm{HAB})+\mathrm{E}$ & -0.060837 & 0.001465 & -41.52 & $1.93 \mathrm{E}-13$ & 0.9937 & $1.93 \mathrm{E}-13$ \\
\hline $\log (\mathrm{GINI})=\beta 0-\beta 1 \log (\mathrm{VERDE})+\mathrm{E}$ & -0.3551 & 0.0247 & -14.37 & $1.78 \mathrm{E}-08$ & 0.9494 & $1.78 \mathrm{E}-08$ \\
\hline $\log (\mathrm{GINI})=\beta 0-\beta 1 \log (\mathrm{AGUA})+\mathrm{E}$ & -0.062673 & 0.001919 & -32.66 & $2.64 \mathrm{E}-12$ & 0.9898 & $2.64 \mathrm{E}-12$ \\
\hline
\end{tabular}

Fuente: Elaboración propia.

Con base en un análisis comparativo entre los coeficientes de la tabla hemos decidido optar por el modelo restringido $\log (\mathbf{G I N I})=$ = $+ß 1 \log (\mathbf{M O V})+\mathbf{E}$ debido a que explica en mayor proporción (99\%) la variabilidad del Coeficiente GINI, captado por la variable independiente MOV (capacidad de atención de pacientes, que tiene el Municipio de Guayaquil a través del programa de Clínicas Móviles), además de presentar mayor nivel de significancia.

\section{Consistencia del modelo $\log (\mathrm{GINI})=ß 0+ß 1 \log (\mathrm{MOV})+\mathrm{E}$}

Existe consistencia en el modelo determinado, observado una relación inversamente proporcional entre la variable explicativa MOV (capacidad de atención de salud en clínicas móviles del Municipio de Guayaquil) y la variable explicada GINI (coeficiente que mide la desigualdad para el cantón Guayaquil), dicho de otra forma en la medida en que aumenta la capacidad de atención de las clínicas móviles, disminuye el coeficiente GINI para la ciudad de Guayaquil.

\section{Significancia del modelo: $\log (\mathrm{GINI})=ß 0+ß 1 \log (\mathrm{MOV})+\mathrm{E}$}

Call:

Im(formula = DATO\$LGINI DATO\$LMOV)

\section{Residuals:}




\begin{tabular}{|c|c|c|c|c|}
\hline Min & 1Q & Median & 3Q & Max \\
\hline-0.092971 & -0.043290 & 0.004446 & 0.054978 & 0.077374 \\
\hline
\end{tabular}

\section{Coefficients:}

\begin{tabular}{|c|c|c|c|c|}
\cline { 2 - 5 } \multicolumn{1}{c|}{} & Estimate & Std. Error & t value & $\operatorname{Pr}(>|\mathbf{t}|)$ \\
\hline (Intercept) & 0.6136 & 0.3666 & 1.674 & 0.12512 \\
\hline DATO\$LMOV & -0.1186 & 0.0283 & -4.190 & $0.00186^{* *}$ \\
\hline
\end{tabular}

$---$

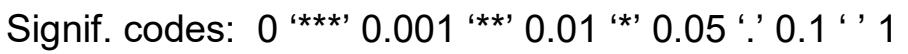

Residual standard error: 0.06283 on 10 degrees of freedom

Multiple R-squared: $0.6372, \quad$ Adjusted R-squared: 0.6009

F-statistic: 17.56 on 1 and $10 \mathrm{DF}, \mathrm{p}$-value: 0.001857

\section{Evaluación del coeficiente:}

La evaluación del valor $P$ nos muestra que con un coeficiente significativo de 0.00186 tenemos evidencia estadística fuerte para rechazar la Hipótesis Nula.

Ho : Máxima probabilidad de cometer un error de tipo I

\section{Análisis de Restricciones:}

F-statistic: 2227 on 1 and 11 DF, p-value: 0.001857

Los coeficientes obtenidos por dicho análisis nos muestran un valor DF de 0.00186 con lo que concluimos que si tenemos un modelo a evaluar 

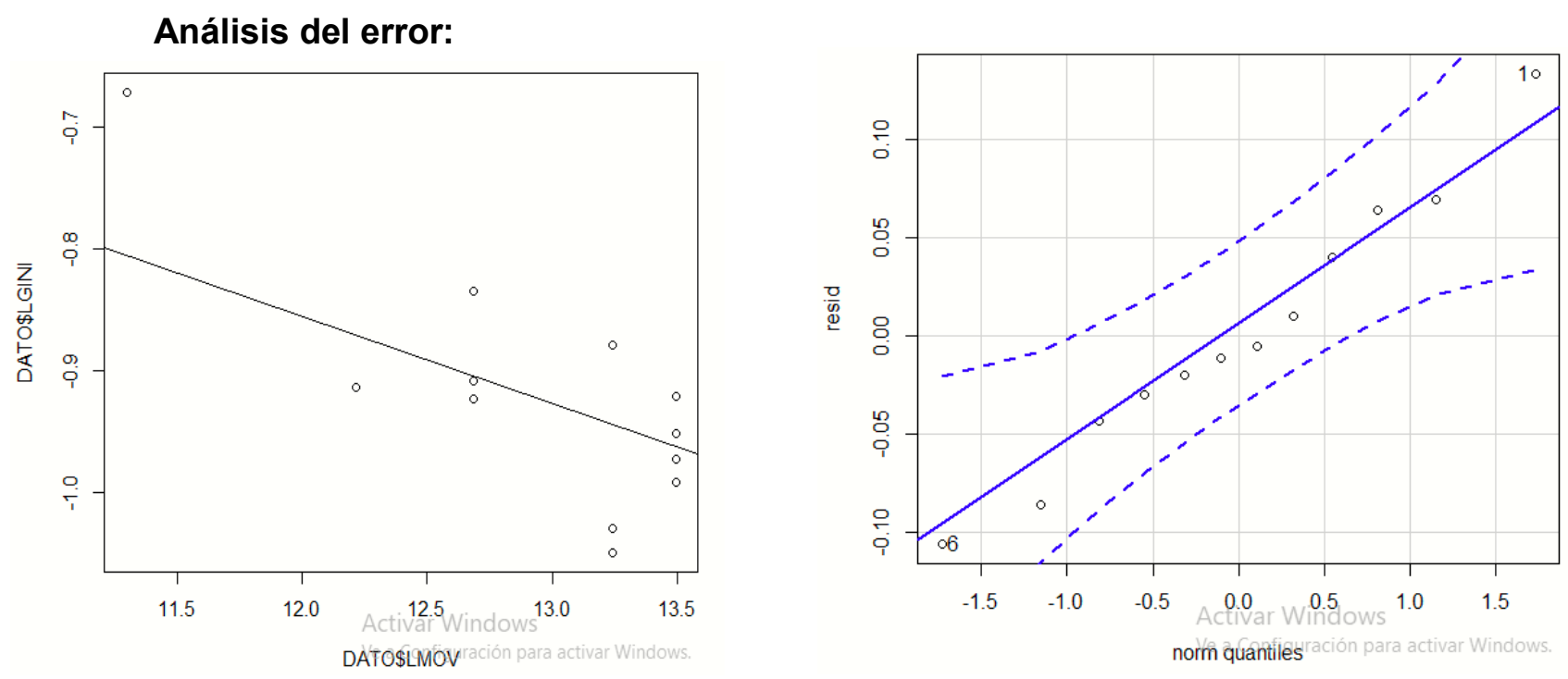

Histogram of resid

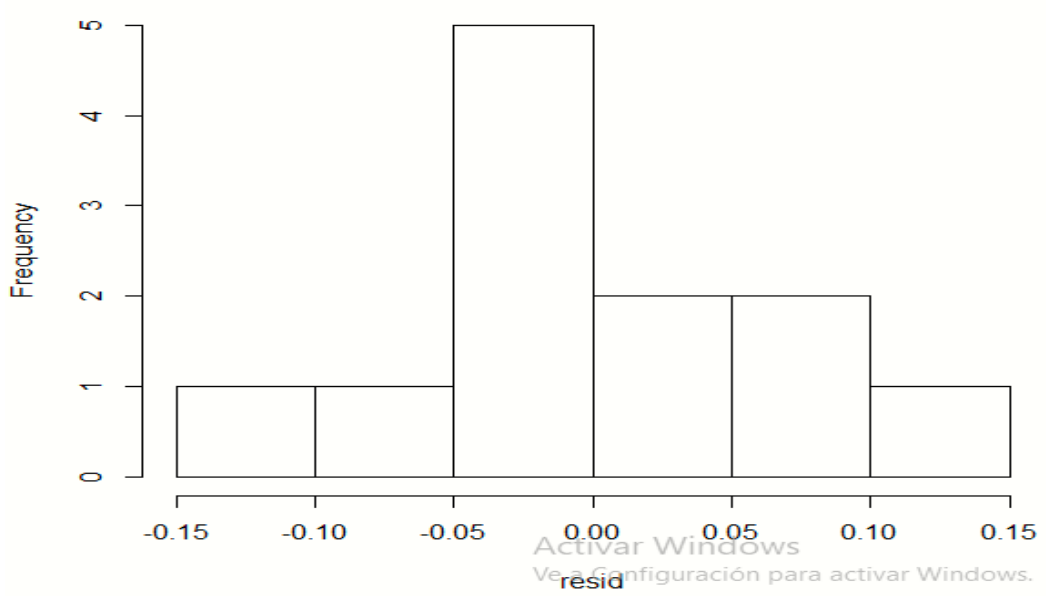

Los gráficos observados muestran que el error sigue una distribución normal.

Interpretación de la variabilidad $\boldsymbol{r}^{2}$

Residual standard error: 0.06283 on 10 degrees of freedom

Multiple R-squared: $0.6372, \quad$ Adjusted R-squared: 0.6009

F-statistic: 17.56 on 1 and $10 \mathrm{DF}, p$-value: 0.001857

El $63 \%$ de la variabilidad de la desigualdad de ingresos de los habitantes de la ciudad de Guayaquil (GINI), logra ser captada por la variable MOV (capacidad de atención en salud de las clínicas móviles del Municipio de Guayaquil).

Interpretación de la forma funcional del estimador ß1 de la variable explicativa MOV 
Considerando el modelo estimado Log-Log cuya forma funcional es ; $\ln (\mathrm{Y})=$ ßo+ $\mathbf{B 1} \ln (\mathbf{X})$ interpretamos que si incrementa la capacidad de atención en salud del Municipio de Guayaquil por medio de las clínicas móviles (MOV) en 1\% entonces el Coeficiente de Desigualdad Guayaquil (GINI) disminuirá en $0.11 \%$ en promedio (ß1\%).

Si ß1 es igual a cero el índice de desigualdad naturalmente aumentará en un $0.6 \%$

\section{Prueba Ressettest:}

RESET test

data: MODELO

RESET $=0.8557$, df1 $=2$, df2 $=8, p$-value $=0.4605$

El resultado expuesto en la prueba ressettest nos indica que existe evidencia estadística fuerte para no rechazar la Hipótesis Nula, esto significa que la forma funcional es lineal.

$\mathbf{H o}=$ La Forma Funcional es Lineal

\section{Prueba Anderson-Darling:}

Anderson-Darling normality test

data: resid

$A=0.34125, p$-value $=0.4303$

El resultado expuesto en la prueba Anderson-Darling muestra evidencia estadística fuerte para no rechazar la Hipótesis Nula, esto significa que los datos siguen una distribución normal

Ho = La Distribución es normal

\section{Prueba BP (Breusch - Pagan):}

studentized Breusch-Pagan test

data: MODELO

$\mathrm{BP}=0.052137, \mathrm{df}=1, \mathrm{p}$-value $=0.8194$

El resultado obtenido en la prueba Prueba BP (Breusch - Pagan) muestra evidencia estadística fuerte para no rechazar la Hipótesis Nula, esto significa que la varianza es constante. Ho $=\mathrm{El}$ error es homecedástico 


\section{Prueba Errores Robustos:}

$\mathrm{t}$ test of coefficients:

\begin{tabular}{|c|c|c|c|c|}
\cline { 2 - 5 } \multicolumn{1}{c|}{} & Estimate & Std. Error & t value & $\operatorname{Pr}(>|t|)$ \\
\hline (Intercept) & 0.613638 & 0.366624 & 1.674 & 0.125123 \\
\hline DATO\$LMOV & -0.118584 & 0.028299 & -4.1904 & $0.001857^{* *}$ \\
\hline
\end{tabular}

Signif. codes: 0 “***) 0.001 “** 0.01 ‘* 0.05 ‘' 0.1 “' 1

Considerando que el modelo no es heterocedástico, la prueba de errores robustos no genera cambios en coeficientes ni en sus valores $P$

\section{Prueba Durbin Watson:}

data: MODELO

$\mathrm{DW}=2.6758, \mathrm{p}$-value $=0.8248$

alternative hypothesis: true autocorrelation is greater than 0

El resultado obtenido en la prueba Durbin Watson muestra evidencia estadística fuerte para no rechazar la Hipótesis Nula, esto significa que hay ausencia de correlación serial, es decir que la capacidad de atención en salud que tiene la Municipalidad de Guayaquil, depende de sí misma y no de otros factores en el tiempo.

Ho = La autocorrelación es 0

Figura 1. Componentes de la serie de tiempo.

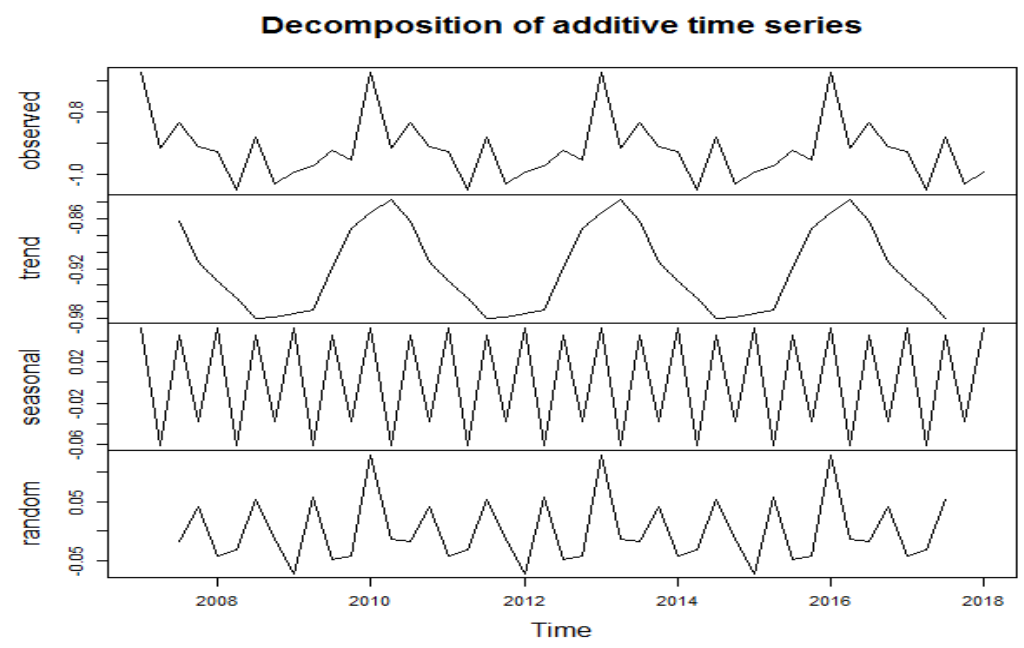

\section{Evaluación de Modelo Autoregresivo}

Call: 
$\operatorname{Im}($ formula $=\mathrm{y} \sim-1+\mathrm{y} \cdot \mathrm{L} 1)$

\section{Residuals:}

\begin{tabular}{|c|c|c|c|c|}
\hline Min & 1Q & Median & 3Q & Max \\
\hline-0.24487 & -0.12939 & -0.01833 & 0.05493 & 0.27704 \\
\hline
\end{tabular}

\section{Coefficients:}

\begin{tabular}{|c|c|c|c|c|}
\cline { 2 - 5 } \multicolumn{1}{c|}{} & Estimate & Std. Error & t value & $\operatorname{Pr}(>|\mathrm{t}|)$ \\
\hline y.L1 & 0.99633 & 0.0223 & 44.52 & $<2 \mathrm{e}-16^{* * *}$ \\
\hline
\end{tabular}

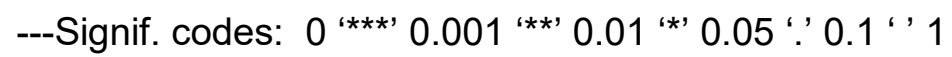

Residual standard error: 0.137 on 43 degrees of freedom

(1 observation deleted due to missingness)

Multiple R-squared: $0.9788, \quad$ Adjusted R-squared: 0.9783

F-statistic: 1982 on 1 and 43 DF, p-value: $<2.2 \mathrm{e}-16$

Los coeficientes obtenidos rechazan la hipótesis nula, lo que significa que la serie no es explosiva, por lo tanto no tiene tendencia

\section{Test de Raíz Unitaria:}

Augmented Dickey-Fuller Test

data: y

Dickey-Fuller $=-3.1997$, Lag order $=3$, p-value $=0.09919$

alternative hypothesis: stationary

El test de raíz unitaria confirma que la serie es estacionaria.

\section{Test Dickey Fuller de la primera diferencia (período anterior):}

Augmented Dickey-Fuller Test

data: d.y

Dickey-Fuller $=-2.494$, Lag order $=3, p$-value $=0.3782$

alternative hypothesis: stationary 
Este test nos indica que se rechaza la Hipótesis Nula, por tanto, la variación del año anterior no incide en el comportamiento del último período.

\section{CONCLUSIONES}

Las desigualdades territoriales son un problema vigente en latinoamerica, por tanto los gobiernos luchan por construir un modelo innovador de política pública que supere los límites de la desigualdad y exclusión social con un enfoque territorial, potenciando las características propias en sinergia con los actores locales.

Por tanto, debido a la creciente tendencia globalizadora, así como la búsqueda del desarrollo del capital intelectual, el territorio pasa a convertirse de un lugar físico para ser habitado, a ser considerado un espacio dónde se despliegan actividades productivas y estratégicas que busca el desarrollo y mejoramiento de las prácticas empresariales a través de la implementación de planes innovadores con la acción de instituciones públicas locales con miras a promover una eficiente gestión social y distribución del ingreso.

En la ciudad de Guayaquil se registran niveles altos de desigualdad social y económica a consecuencia del incremento poblacional debido a las corrientes migratorias tanto a nivel nacional e incluso internacional, lo cual ha generado un incremento en la población que habita en sectores urbano marginales, muchos de estos carentes de acceso a servicios básicos.

De este modo, el presente trabajo de investigación determinó el impacto de la gestión municipal en la disminución del índice de desigualdad medido por el coeficiente GINI en el cantón Guayaquil, analizando un conjunto de variables que en forma de política pública, son implementadas por la administración municipal tales como: dotación de clínicas móviles, cobro de impuestos prediales, tasas de habilitación, asignación de metros cuadrados de áreas verdes por habitante y cobertura del servicio de agua potable, todas consideradas iniciativas que aportan a la disminución de la desigualdad. El período evaluado comprende a partir del año 2007 al 2018 con datos extraídos de las paginas oficiales del Banco Central del Ecuador, Municipio de Guayaquil e Interagua. 
Mediante el planteamiento de un modelo empírico de regresión lineal hemos propuesto un análisis econométrico de la desigualdad de ingresos (medido por el coeficiente de Gini) en la ciudad de Guayaquil, en donde se pudo dar validez del impacto de una de las variables propuestas en el modelo; como fue el caso de la variable independiente Clínicas Móviles (MOV). Con respecto a las otras variables propuestas, concluimos que presentaron problemas de colinealidad, es decir, que tenían una fuerte correlación entre sí dentro del modelo regresivo desarrollado.

Por lo tanto, se pudo determinar que la variable independiente Clínicas Móviles (MOV) podía explicar de mejor manera el impacto generado en la disminución del coeficiente GINI en la ciudad de Guayaquil, ya que mostró consistencia y significancia en el modelo de regresión propuesto. Los gráficos resultantes del modelo de regresión (Histogram-Quantiles), el análisis de error y la prueba ressettest permiten observar que los datos siguen una distribución normal y a su vez determinan linealidad, mientras. De la misma forma se observa resultados positivos en la variabilidad $r^{2}$, en donde se determina que 63\% de la variabilidad de la desigualdad de ingresos de los habitantes de la ciudad de Guayaquil (GINI), logra ser captada por la variable MOV (capacidad de atención en salud de las clínicas móviles del Municipio de Guayaquil).

Concluimos también que el modelo estimado, cuya forma funcional es ; $\ln (\mathbf{Y})=$ ßo+ß1 $\ln (\mathbf{X})$ nos permite interpretar que si incrementa la capacidad de atención en salud del Municipio de Guayaquil por medio de las clínicas móviles (MOV) en 1\% entonces el Coeficiente de Desigualdad Guayaquil (GINI) disminuirá en $0.11 \%$ en promedio (ß1\%).

La prueba Anderson-Darling mostraron que los datos siguen una distribución normal, mientras La prueba Breusch - Pagan expone que la varianza es constante, por consiguiente el modelo presentaba homocedasticidad, es decir que su variable explicativa llegó a ser constante a lo largo de la observación, considerando que el modelo no es heterocedástico, la prueba de errores robustos no genera cambios en coeficientes ni en sus valores $P$.

El resultado obtenido en la prueba Durbin Watson muestra evidencia estadística fuerte para no rechazar la Hipótesis Nula, esto significa que hay ausencia de correlación serial, es decir que la capacidad de atención en salud que tiene la Municipalidad de Guayaquil, depende de sí misma y no de otros factores en el tiempo. 
Con respecto a las series de tiempo se llegó a la conclusión que dicho modelo posee estacionalidad entre sus datos, es decir que la variabilidad del período anterior no incide en los cambios que vayan a tener en el último período, es decir que no siguen una tendencia a través del tiempo.

Finalmente se concluye que los resultados obtenidos en el modelo econométrico desarrollado, confirman la hipótesis planteada en este trabajo, mostrando a través de los coeficientes obtenidos, que la variabilidad en la cobertura de atención de salud a través de clínicas móviles, tiene incidencia en la variabilidad del índice de desigualdad GINI, por tanto, explica el fenómeno estudiado en este proyecto de investigación

\section{REFERENCIAS BIBLIOGRÁFICAS}

Alemán, A., M. A. (2019). Desigualdades territoriales y desarrollo local. Consideraciones para Cuba. Revista Economía y Desarrollo (Impresa), 138(2).

Asamblea Nacional de Ecuador. (2010). COOTAD. Quito: Registro Oficial S 303.

Boisier, S. (1988). Palimpsesto de las regiones como espacios socialmente construidos. Área de planificación y política regional CEPAL, 54-55.

Bonnin, C.-J. (1812). Principios de administración pública. París.

Casas, E. C. (2018). Casas, E. C. (2018). El desarrollo económico local, el enfoque de desarrollo humano y la economía social y solidaria al servicio de la persona. REVISTA PERSPECTIVA (19) 2, 227.

Castells, M. (1998). La era de la información. Alianza.

Correa, L. E. (2016). Descentralización y desarrollo regional en Chile. Polis , 3.

Garofoli, G. (1986). Modelos Locales de Desarrollo. Lecciones de la experiencia italiana.

Marx, C. (1968). Notas críticas al artículo "El Rey de Prusia y la reforma social por un prusiano". México: Grijalbo.

Molina, J. E. (2015). La Descentralización en Colombia, ¿realidad o espejismo? consideraciones sobre la actualidad del proceso. CEA, 1(1), 75-64.

Muñoz, J. G. (2015). Economías solidarias y territorio: hacia un análisis desde la complejidad. Otra economía 9(17), 123-135.

Stein, L. (1897). La cienca de la administración pública. Turín. 
Stiglitz, J. E. (2012). El precio de la desigualdad. USA: Taurus.

Vásquez Barquero, A. (1999). Desarrollo, redes e innovación. El desarrollo local en los tiempos de globalización. Pirámide.

Vázquez, M. C. (2015). La descentralización: Un caminos al desarrollo territorial. Consorcio de Gobiernos Autónomos Provinciales del Ecuador, 61-76.

Veiga, D., \& Rivoir, A. L. (2004). Desigualdades sociales en Uruguay: desafíos para las políticas de desarrollo. Montevideo. 